\title{
ESTRO ACROP: Technology for precision small animal radiotherapy research: Optimal use and challenges
}

\author{
Frank Verhaegen a,*, Ludwig Dubois ${ }^{a}$, Stefano Gianolini ${ }^{b}$, Mark A. Hill ${ }^{c}$, Christian P. Karger ${ }^{\text {d,e }}$, \\ Kirsten Lauber ${ }^{\mathrm{f}}$, Kevin M. Prise ${ }^{\mathrm{g}}$, David Sarrut ${ }^{\mathrm{h}}$, Daniela Thorwarth ${ }^{\mathrm{i}}$, Christian Vanhove ${ }^{\mathrm{j}}$, \\ Boris Vojnovic ${ }^{c}$, Robert Weersink ${ }^{\mathrm{k}}$, Jan J. Wilkens ${ }^{1}$, Dietmar Georg ${ }^{\mathrm{m}}$
}

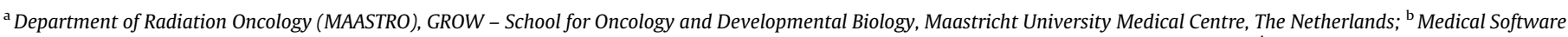

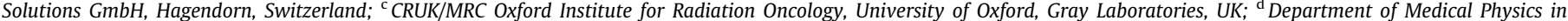

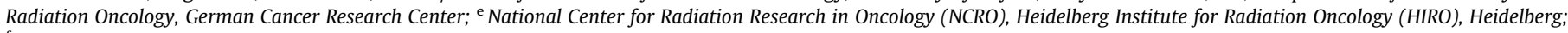

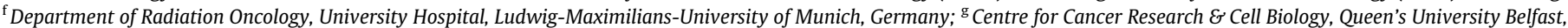

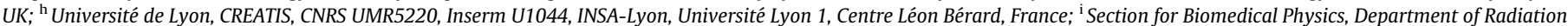

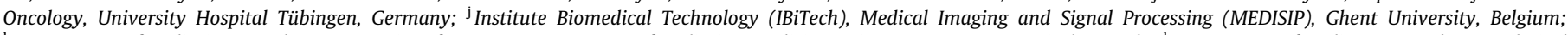

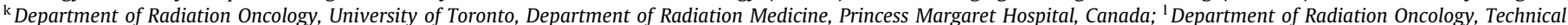

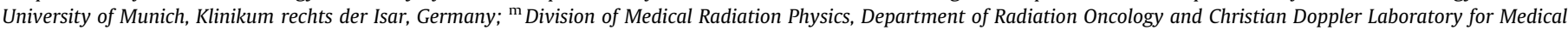
Radiation Research for Radiation Oncology, Medical University of Vienna, Austria
\end{abstract}

\section{A R T I C L E I N F O}

Article history:

Received 18 October 2017

Accepted 21 November 2017

Available online $\mathrm{xxxx}$

\section{Keywords:}

Radiotherapy

Precision

Small animal

Image guided

Irradiation

\begin{abstract}
A B S T R A C T
Many radiotherapy research centers have recently installed novel research platforms enabling the investigation of the radiation response of tumors and normal tissues in small animal models, possibly in combination with other treatment modalities. Many more research institutes are expected to follow in the coming years. These novel platforms are capable of mimicking human radiotherapy more closely than older technology. To facilitate the optimal use of these novel integrated precision irradiators and various small animal imaging devices, and to maximize the impact of the associated research, the ESTRO committee on coordinating guidelines ACROP (Advisory Committee in Radiation Oncology Practice) has commissioned a report to review the state of the art of the technology used in this new field of research, and to issue recommendations. This report discusses the combination of precision irradiation systems, small animal imaging (CT, MRI, PET, SPECT, bioluminescence) systems, image registration, treatment planning, and data processing. It also provides guidelines for reporting on studies.

(C) 2017 The Authors. Published by Elsevier Ireland Ltd. Radiotherapy and Oncology xxx (2017) xxx-xxx This is an open access article under the CC BY-NC-ND license (http://creativecommons.org/licenses/by-nc-
\end{abstract}

nd/4.0/).

Translational and radiobiological research is currently undergoing a revolution due to two key developments: (1) the availability of advanced tumor models with more clinically relevant tumor environments, and (2) the availability of technology that allows precise radiation targeting, using onboard integrated imageguidance which can mimic clinically advanced radiotherapy treatments in an experimental setting (Fig. 1). Such precision irradiators facilitate studies that explore temporal and spatial dose modulation, and novel combinations of radiation with other therapeutic or protective agents, both for radiation response of tumors and normal tissues. The aim of these studies is then to generate results that can be translated more rapidly into clinical trials, benefitting patients [1,2].

These new technologies, for small animal research, bring an extensive range of challenges that need careful assessment to

\footnotetext{
* Corresponding author.
}

allow their future optimal use for translational research. Specific challenges include: (1) What are the key technologies required to downscale clinical treatments into small animal models? (2) How to deal with target motion? (3) Which imaging modalities should be integrated into the radiation platforms? (4) What are the optimal irradiation margins? (5) What is the accuracy and precision of small field dosimetry? (6) Which methods should be developed to verify the dose distribution? (7) Which imaging modalities should be used for treatment planning, given the evolving clinical scenarios? (8) What is the difference between high and low-energy photon irradiation?

In the framework of ACROP (ESTRO's Advisory Committee in Radiation Oncology Practice), the ESTRO committee coordinating guidelines, this newly established writing committee's mandate is to review and discuss the state of the art in this new field of research, covering the technology [3,4] currently available for image-guided small animal radiation research such as precision 


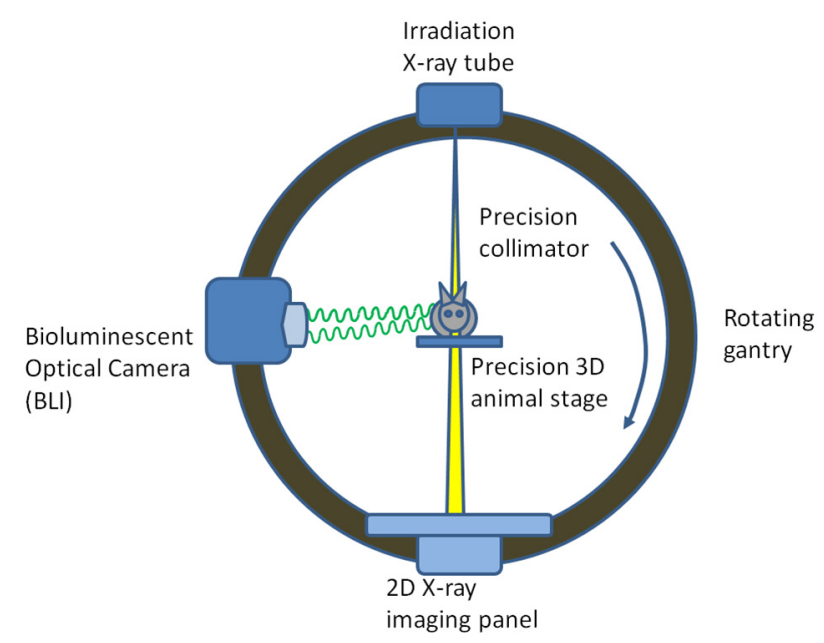

Fig. 1. Generic sketch of an image-guided precision irradiator. In this implementation radiation can be administered in an arc, and cone beam CT imaging is performed by a rotating gantry mounted X-ray panel. In other implementations the animal may rotate to acquire a CT image. In this example there is also a bioluminescent camera mounted on the gantry, which allows capturing optical photons emitted by labeled structures in the animal. Once the target volume has been identified, a collimated X-ray beam is used to deliver the therapeutic dose, either in the form on multiple stationary beams or a continuous arc.

irradiation systems, imaging (CT, MRI, PET, SPECT, bioluminescence) systems, image registration, treatment planning, and data processing. Finally, animal radiation research with light ion beams is also briefly addressed, although the latter is usually not performed with dedicated small animal beams, but rather with specialized setups at ion beams for radiotherapy.

Basically, three categories of users of radiation in animal studies can be identified: ones that need a known arbitrary but reproducible radiation dose (e.g. to study synergistic effects), ones that need a precise dose or range of doses (e.g. to establish a dose-response curve) and ones that need a modulated dose distribution (in time and/or space). This review is intended to be a first step toward aiding users to define optimal studies and toward guiding developers with respect to future improvements, with a strong focus on the latter two categories.

\section{Commissioning and operating precision irradiators}

Preclinical research platforms which have been developed, commercially or otherwise, are summarized in Table 1 . The precision and accuracy of the novel irradiators are critically dependent on how well they have been commissioned and the subsequent quality control [5-7].

Table S1 lists the main issues that need to be considered during commissioning. Ideally commissioning should be performed by a medical/radiation physicist, who has expertise in radiation source commissioning using suitable phantoms. For inexperienced users, commissioning may be done by the manufacturer, but in this case it is recommended that a report is provided with the raw data and any processed data. A key part of this is absolute dosimetry using standardized protocols (e.g. [18-20]) and calibrated equipment traceable to a primary standard. To ensure continuous accuracy it is essential that regular quality assurance measurements are made, this may vary from daily for critical but simple output checks, to monthly for more detailed checks. If real-time dosimetry is desired, small radiation detectors such as mosfets or optical fibers may be considered, or the onboard X-ray imager may be used to verify the treatment [21].

In practice the dose distribution is dependent on many factors, including the photon spectrum, irradiation geometry, the composition and geometry/anatomy of the animal being irradiated and surrounding scattering/attenuating materials. Each of these needs to be adequately described along with the details of the dose measurement/calculation. In addition to the physical dose, the ultimate biological response can also depend on factors such as dose rate and radiation quality [22,23]. Unfortunately, the experimental setup, dosimetry and exposure details are often inadequately reported in the scientific literature, emphasizing the necessity to develop standard operating procedures [24,25] with the key issues summarized in Table S2. This information is required to assess the quality and limitations of preclinical data, to ensure that any effects observed are not artifactual, and thus to evaluate the translational potential of the data and hypotheses generated for the development of clinical trials [26].

Recommendations for reporting studies can be found in Table 2.

\section{Treatment planning systems}

Treatment planning systems (TPS) for small animal irradiators face several challenges [27]. Commonly, the target volume is small, rarely exceeding a cubic $\mathrm{cm}$. Thus, irradiation is preferentially performed with about $225 \mathrm{kV}$ instead of MV photon beams to avoid large dose-build-up effects at medium interfaces and wide penumbrae [4]. Together with the small field sizes, this renders calculation models implemented in clinical MV treatment planning systems (TPS) unsuitable, since apart from Compton scatter the tissue-dependent photo-electric effect needs to be considered, and the resulting inaccuracies for small beams would be unacceptable [27]. Different calculation models have been implemented in small animal irradiators with Monte Carlo simulation and Superposition-Convolution being the most prominent ones [4,6,21,28-31] (Table 3).

The workflow of preclinical treatment planning generally mimics clinical radiotherapy (Fig. 2). The main difference is that treatment planning and dose administration are performed in one session while the animal is under anesthesia. Multi-modal functional or molecular imaging is also available for preclinical treatment planning [32], see also subsection on imaging devices.

Despite all technical advances in preclinical treatment planning, several critical issues still remain. As such, commissioning of the TPS represents major challenges (Table S3), particularly for very small beams [6,7,33]. Moreover, photon scatter is poorly studied for narrow beams of $\mathrm{kV}$ energies in small animals. It may interfere with CBCT imaging quality as well as with accuracy of dose calculation [7]. The procedure of tissue segmentation also involves several open issues, including the aspect of arbitrariness and the

Table 1

Examples of commercial and non-commercial image-guided small animal irradiators.

Commercial

SARRP system (Xstrahl Ltd, UK) [8-10];

X-RAD 225CX (Precision X-ray Inc., USA) [11]
Non-commercial research machines

Brachytherapy-based system using an Ir-192 source [12] System based on the GE micro-CT scanner $[5,13,14]$ $\mathrm{X}$-ray image guidance system [15]

Carbon nanotube [16]

SAIGRT system [17] 
Table 2

Requirements for specification and reporting of small animal irradiation with particle beams.

\begin{tabular}{|c|c|}
\hline \multicolumn{2}{|c|}{ Common requirements for studies with photons and particles (See also Table S1) } \\
\hline $\begin{array}{l}\text { Irradiation modality } \\
\text { Absorbed dose } \\
\text { Field size } \\
\text { Margins } \\
\text { Treatment fields } \\
\text { Optimization }\end{array}$ & $\begin{array}{l}\text { Photon energy, filtration, } \mathrm{kVp} \text {, half value layer, particle type and particle energy } \\
\text { Dose prescription point or volume, 1-2-3D dose distribution }\end{array}$ \\
\hline \multicolumn{2}{|c|}{ Specific requirements for particle beam studies } \\
\hline $\begin{array}{l}\text { Depth dose profile } \\
\text { Position of the target } \\
\text { tissue within the } \\
\text { beam }\end{array}$ & $\begin{array}{l}\text { Pristine Bragg peak: energy and range of primary particles } \\
\text { Spread Out Bragg Peak: range and depth of the proximal and distal edge of high-dose region }\end{array}$ \\
\hline Linear Energy Transfer & $\begin{array}{l}\text { Track-averaged or dose-averaged LET or another beam quality specifier including range of LET } \\
\text { for extended targets }\end{array}$ \\
\hline Setup & Detailed information on of setup and range adjustment (collimators, range shifters) \\
\hline SOBP & $\begin{array}{l}\text { Specification whether the SOBP was optimized in terms of absorbed or RBE-weighted dose. If the latter, } \\
\text { which RBE-model and model parameters were used, and the type of photon reference radiation }\end{array}$ \\
\hline
\end{tabular}

Table 3

Comparison of dose calculation models currently implemented in treatment planning systems of small animal precision irradiators.

\begin{tabular}{|c|c|c|}
\hline & Superposition-Convolution dose calculation & Monte Carlo simulation \\
\hline Principle & $\begin{array}{l}\text { Local photon energy fluence derived from the primary energy fluence } \\
\text { emanating from the photon source multiplied by energy absorption } \\
\text { coefficient gives total energy released to matter (terma). Ray-tracing } \\
\text { with inclusion of } 1 \text { st order Compton scatter }\end{array}$ & $\begin{array}{l}\text { Modeling of particle transport, interactions with other particles and } \\
\text { electromagnetic fields, and production of secondary particles based } \\
\text { on cross sections and transport theories. Consideration of Compton } \\
\text { scatter and photo-electric effect }\end{array}$ \\
\hline Input & $\begin{array}{l}\text { Photon spectrum or phase space file obtained from separate Monte } \\
\text { Carlo simulation. Geometry and materials are defined from CT } \\
\text { images. }\end{array}$ & $\begin{array}{l}\text { Photon spectrum or phase space file obtained from separate Monte } \\
\text { Carlo simulation. Geometry and materials are defined from CT } \\
\text { images. }\end{array}$ \\
\hline Dose reporting & $\begin{array}{l}\text { Dose-to-water-in-medium, convertible into Dose-to-medium-in- } \\
\text { medium }\end{array}$ & Dose-to-medium-in-medium and dose-to-water-in-medium \\
\hline Limitation & $\begin{array}{l}\text { Inaccuracies in non-water media with dose discontinuities (lung, } \\
\text { bone) }\end{array}$ & $\begin{array}{l}\text { Computing time (getting less important with new and faster } \\
\text { computers) }\end{array}$ \\
\hline $\begin{array}{l}\text { Example treatment } \\
\text { planning system }\end{array}$ & 3D Slicer-based MuriPlan [40] & MatLab-based SmART-Plan [41] \\
\hline
\end{tabular}

"From July 2017 onward this is replaced by SmART-ATP (SmART Scientific Solutions BV, Maastricht, the Netherlands).

question how many tissue types are needed for proper dose calculation accuracy [34-36]. Dose reporting is another concern. Doseto-water-in-medium and dose-to-medium-in-medium are two completely different quantities which are currently used in parallel, and it is so far entirely unclear which one correlates better with the biological effects of ionizing irradiation. Both should be available but conversion from one to the other introduces uncertainties [27]. As small animal planning will become more complicated, beam optimization will be needed [37,38]. Finally, intrairradiation motion is a largely understudied aspect in preclinical radiotherapy which may be addressed by time-resolved imaging, gated beam delivery, and/or target tracking [39,91].

\section{Image guidance systems}

In the following sections we will discuss the commissioning and operation of various small animal imaging methods for precision irradiation. A recent report covered animal imaging quality control [42]. Table S4 gives an overview of guidelines and future development for the various imaging modalities.

\section{Computed tomography (CT)}

$\mathrm{CT}$ is a pre-requisite for heterogeneity corrections in dose calculation $[27,43]$ for treatment planning. CT images in DICOM format can be visualized using common research software platforms (e.g. MatLab, Osirix, 3D Slicer). There are different technological solutions for preclinical CT $[4,44]$. Dedicated micro-CTs have a rotating X-ray tube and imaging panel configuration. Modern animal research platforms use either a fixed animal and rotating X-ray tube/imager or vice versa, resulting in a cone beam CT (CBCT) scan. Typical X-ray energies are between 35 and $90 \mathrm{keV}$. Amorphous silicon imaging panels are standard, and image reconstruction is based on the Feldkamp algorithm [45].

The technical realization and the underlying projection geometry vary and have a direct impact on the imaging parameters. There are geometric aspects and accuracy requirements for irradiation units with integrated CT imaging options, i.e. flex-correction maps during imaging and irradiation need to be determined and verified during regular quality assurance as well as Hounsfield Unit (HU) calibration [7,42]. The mechanical hardware accuracy is reported to be below $100 \mu \mathrm{m}$ [15]. The typical image resolution (pixel dimension) for CBCT is in the range of $100-200 \mu \mathrm{m}$, and even lower for micro-CTs. The modulation transfer function (MTF) of CBCT and micro-CT at the $10 \%$ level are around $1 \mathrm{~mm}^{-1}$ and $2 \mathrm{~mm}^{-1}$, respectively. To determine spatial resolution, contrast, etc. dedicated phantoms are required [46].

Imaging and treatments in the keV energy range imply the availability of dedicated dosimetry equipment [18]. Typical imaging doses to small animals are around $0.3 \mathrm{~Gy}$ or even higher for micro-CT [47]; and need to be considered in longitudinal studies especially for non-tumor tissue. Technical solutions for respiratory motion management have been reported [48], and next generation systems are expected to offer dual energy options [49]. 


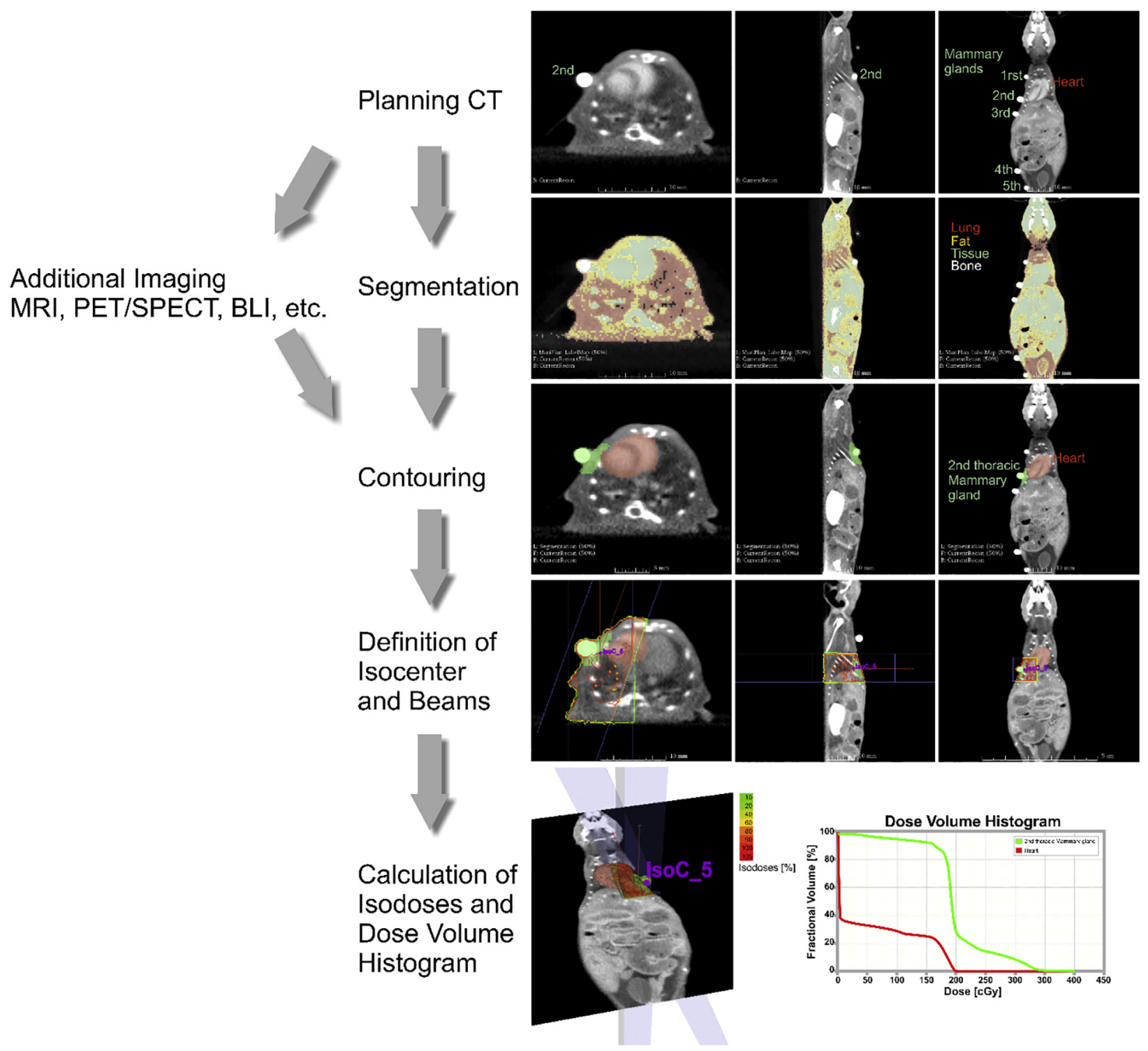

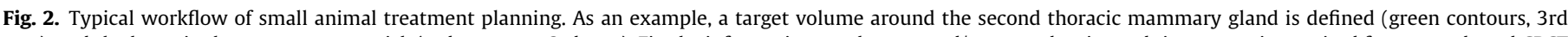

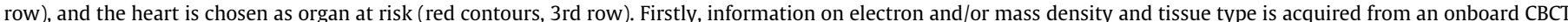

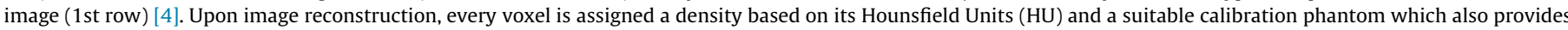

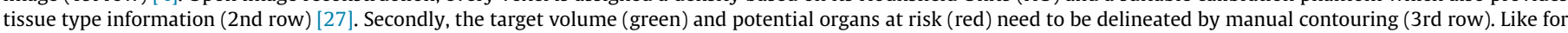

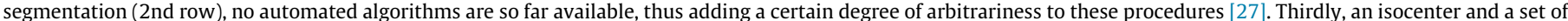

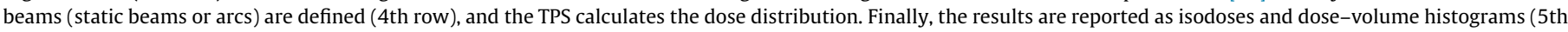
row), and the delivery settings are transferred to the irradiator.

\section{Positron emission tomography (PET) and single photon emission tomography (SPECT)}

Treatment planning for preclinical precision irradiators is predominantly based on CT imaging. Additional information from nuclear medicine based imaging techniques, i.e. PET and SPECT, can be used for (sub-)target volume definition in dose painting strategies, for response assessment in longitudinal studies, or in the context of imaging and therapeutic biomarker research [50-53].

Image quality in SPECT/PET is mainly determined by spatial resolution and sensitivity. Recent SPECT cameras obtain submillimeter spatial resolution using a multi-pinhole multidetector design and can obtain sensitivities in the order of $0.1 \%$. Preclinical PET cameras are downscaled models of human systems, usually with an axial field-of-view that enables whole-body mouse imaging. Spatial resolution in PET is around $1 \mathrm{~mm}$ and sensitivity can be very high (14\% is reported). Although SPECT offers several advantages in study flexibility, radiochemistry complexity, tracer half-lives and cost, PET is currently the most sensitive and quantitatively accurate nuclear imaging modality [54].

Because SPECT/PET are considered quantitative imaging techniques, image degrading effects, such as photon attenuation and scatter should be corrected during the reconstruction process. The reconstructed voxel size should be at least three times the spatial resolution of the imaging system to minimize partial volume effects [55]. Finally, SPECT/PET requires the injection of radioactive tracers and cross-calibrations between SPECT/PET camera and a dose calibration is required for accurate quantification [42,53]. This requires imaging dedicated phantoms with a known activity concentration. The same can be used to assess longitudinal quantitative accuracy of the scanner by comparing the known activity in the phantom with that in the reconstructed images [42]. Tracer 
volumes in small animals are limited, often requiring high activity concentrations and, therefore, careful measurement of the injected activity is crucial for accurate quantification.

\section{Magnetic resonance imaging (MRI)}

The clinical use of MRI is rapidly growing, i.e. for MR based treatment planning including dose calculation, and multiparametric MRI for tissue characterization and response assessment. This trend is based on advantages such as a superior soft-tissue contrast compared to CT and imaging without dose burden, which also impacts pre-clinical research [56-59]. Main disadvantages of MRI are the relatively long acquisition times and its implication on animal anesthesia, the high cost of an MRI scanner and high operational costs.

In the standard irradiation setting, MRI scans are not directly used for dose calculations, but a registered CT/MRI dataset provides the necessary information for targeting (MRI) and for dose calculations (CT) [60]. Geometric aspects including distortion corrections, scanner calibration and image registration need to be considered, for which dedicated phantoms exist $[42,61,62]$.

An MRI-only based workflow for radiotherapy planning becomes feasible with the introduction of dedicated MRI sequences, such as Ultra Short Echo time (UTE) and Zero Echo time (ZTE), where bony structures become visible [57]. For this, a common coordinate system between MRI images and micro-irradiator space is needed, e.g. via $3 \mathrm{D} / 3 \mathrm{D}(\mathrm{MR} / \mathrm{CT})$ registration or using $2 \mathrm{D} / 3 \mathrm{D}$ image registration involving digitally reconstructed radiographs (DRRs).

Pre-clinical MR imaging is performed on dedicated micro-MR systems, which often have ultra-high field strength, or on clinical high field strength systems (i.e. 3T/7T) for which dedicated micro-coils can achieve the high-spatial resolution [52,63]. The high field strengths imply safety aspects. The resolutions of micro-MR units are below $100 \mu \mathrm{m}$ and the ultrahigh fields facilitate spectroscopy research. However, sequences and acquisition protocols used for pre-clinical MRI need to be optimized toward 3D imaging to cover the whole tumor volume and to minimize distortion to guarantee geometric accuracy of the data.

\section{Bioluminescence imaging (BLI)}

For small animal radiotherapy BLI promises improved targeting of tumors not visible on other modalities [64-66], as well as longitudinal response-monitoring. While the BLI signal is weak, there is no background and so it is able to detect as few as 1000 cells injected in an animal. Also, it has a large dynamic range (several orders of magnitude) without delivering any radiation dose $[67,68]$.

A BLI signal can be acquired using non-contact optical imaging or with optical fibers in contact with the tissue [69,70]. BLI collected at multiple wavelengths can aid in target reconstruction, since the signal is wavelength dependent (due to variations in tissue absorption and scattering) [71].

While acquiring BLI images is straightforward, 3D source reconstruction remains a challenge. With multiple techniques under investigation, solutions that allow radiation targeting and response monitoring can be expected in the near future.

Center-of-mass targeting using BLI is possible [72], but radiation targeting requires the 3D geometry of the tumor. However, the resolution and detection depth are limited by scattering of optical photons and the limited knowledge of tissue optical properties [73]. The main 3D reconstruction needs (and limitations to accurately achieving outcomes) are:

(a) a model of the surface and locations of detection points [74,75] (using CBCT [76] or optical scanning)

(b) the location/geometry and optical properties of major organs (requires segmentation on CBCT images; optical properties either measured in situ using diffuse optical tomography [77-80] or taken from library values [81])

(c) calculation of light propagation (using analytical $[73,80]$ or Monte Carlo methods [77,78])

(d) accurate reconstruction algorithm [78,79,81,82] (ill-posed inverse problem)

Together, these steps take considerable time (10-20 min per mouse) that would severely reduce throughput on a system. The process speed for radiation targeting could be improved using accurate auto-segmentation or BLI could be performed at a separate session prior to treatment with the target transferred to treatment images using deformable registration.

\section{Registration of different imaging modalities}

To fully exploit the high precision of small animal image-guided radiotherapy the positioning error of the target has to be minimized. This may require registration of information from various images, possibly from different modalities, usually to a reference CBCT image. This can be done manually or ideally by software to provide spatial registration of the two images by optimization of a 'goodness-of-alignment' metric using appropriate features. Both rigid transformations (only translation and/or rotation) and nonrigid transformations may be needed to allow for changes in size and/or shape of sub-volumes. Although potentially providing better registration, care must be taken when employing the latter to ensure deformations are realistic. Relevant information that should be reported is detailed in Table S2 Additionally, non-rigid registration can be used in longitudinal studies between the initial CBCT image and subsequent scans. This can be used to follow accurately the deformation of organ contours [83].

Depending on which part of the body is being studied, movements may be a major limiting factor to using co-registered images for targeting. While the anatomy of the head remains relatively immobile, significant motion is possible within the abdomen and thorax with time and as a result of handling. In these cases it is essential that procedures are in place to minimize movement between initial imaging and subsequent beam delivery. Recommendations to help ensure accuracy of registration and minimize the effect of movement are summarized in Table S5.

Table 4

Data sets and standards for various preclinical and clinical fields.

\begin{tabular}{|c|c|c|}
\hline Domain & Data set & Applicable standard \\
\hline Molecular and clinical data & The Cancer Genome Atlas (TCGA) molecular and clinical data & $\mathrm{N} / \mathrm{A}$ \\
\hline Clinical imaging & The Cancer Imaging Archive (TCIA) in vivo imaging data & DICOM \\
\hline Pre-clinical & Small animal models & $\begin{array}{l}\text { Suppl 187: Preclinical Animal Imaging Acquisition Context of the } \\
\text { DICOM standard exists but has not yet been adopted }\end{array}$ \\
\hline Digital pathology & caMicroscope & DICOM is applicable but has not yet been adopted \\
\hline All & Annotations and markup on images & $\mu \mathrm{AIM}$ is in development \\
\hline
\end{tabular}




\section{Digital data processing}

In the medical field data standards have been used for many years allowing for standardized storage and easy exchange between different systems and different clinics. In medical imaging, the DICOM standard (Digital Imaging and Communication in Medicine, [84]) was set up to address this and for clinical applications today the DICOM standard is well established among medical imaging equipment vendors and healthcare IT organizations. In the field of preclinical radiobiology and trials, however, far less effort has been put in data standardization, which has hampered data sharing and leveraging results across studies and institutes. Imaging-based cancer research is in the early phase of an integrative-biology revolution making the creation of more robust tools crucial for data interoperability across several domains like genomics (and other omics), diagnostic imaging, and digital pathology (Table 4). The newly available image-guided precision irradiation research platforms present a unique opportunity to develop data standards and powerful data analysis tools that will enable more efficient data extraction and data processing.

Indeed, some of the modern small animal platforms and treatment planning systems already support the DICOM standard for imaging and the DICOM-RT objects (RT Image, RT Structure Set, RT Plan, RT Dose,...). Recent efforts by the DICOM working group 30 have led to a DICOM standard Supplement 187 defining usecases and templates for storage of information related to acquisition of small animal images during preclinical research ([85], Table S6). A common informatics infrastructure for small animal research must also provide researchers with a data warehouse combining various sources of data for easy storage and retrieval of large amounts of information such as pathology, genomics, histology, experimental designs, etc. In addition, a modern software environment for small animal studies should provide analysis tools that can be used to directly mine data from multiple high-volume information repositories, creating a foundation for research and translation of the results of animal studies into clinical trials.

\section{Particle beam studies}

As dedicated small animal irradiation devices are available only for photons, particle irradiations of animals are currently performed in clinical or experimental facilities [86-89]. Also, current TPS are usually not intended to be used for small irradiation fields and integrated imaging devices are mostly not available or not suitable for small animal irradiations with particles except in rare cases [90]. This compromises dosimetric and geometric irradiation accuracy. Together with limited experience with particle beams, this may affect the results and complicate inter-comparison of data between different facilities.

Many requirements for specification and reporting of precision small animal particle irradiations are similar to those of photons, however, particle irradiations require specification and reporting of several additional parameters (Table 2). When using a clinical TPS, one should be aware of the potentially limited accuracy of the predicted absolute dose and lateral field borders. In this respect, verification measurements by pinpoint ionization chambers and radiochromic films may be necessary [87]. When comparing results with photon experiments, the use of identical dose prescription points, dose verification methods and field sizes has to be assured.

In general, small animal irradiation studies should take care that there is a close link to clinical particle beam protocols to assure that results replicate patient exposures. As it is unlikely that there will be many dedicated small animal irradiation facilities in the near future, researchers are encouraged to develop and install dedicated TPS and imaging devices for small animal irradiations at their clinical or experimental beam lines to improve dosimetric and geometric accuracy [90].

\section{Conclusions}

It is strongly recommended to develop protocols and guidelines to use the novel preclinical radiation research platforms to maximize their impact on translation of radiotherapy research into the clinic. This report provides an overview of the relevant technology issues to consider.

\section{Disclaimer}

ESTRO cannot endorse all statements or opinions made on the guidelines. Regardless of the vast professional knowledge and scientific expertise in the field of radiation oncology that ESTRO possesses, the Society cannot inspect all information to determine the truthfulness, accuracy, reliability, completeness or relevancy thereof. Under no circumstances will ESTRO be held liable for any decision taken or acted upon as a result of reliance on the content of the guidelines.

The component information of the guidelines is not intended or implied to be a substitute for professional medical advice or medical care. The advice of a medical professional should always be sought prior to commencing any form of medical treatment. To this end, all component information contained within the guidelines is done so for solely educational and scientific purposes. ESTRO and all of its staff, agents and members disclaim any and all warranties and representations with regards to the information contained on the guidelines. This includes any implied warranties and conditions that may be derived from the aforementioned guidelines.

\section{Conflict of interest}

The authors declare that they have no competing interests. None of the authors has any financial and personal relationships with other people or organisations that could inappropriately influence (bias) this work.

\section{Acknowledgements}

The authors would like to thank Dr Julia Mannheim and Dr Lucas Persoon for comments on the manuscript.

\section{Appendix A. Supplementary data}

Supplementary data associated with this article can be found, in the online version, at https://doi.org/10.1016/j.radonc.2017.11. 016.

\section{References}

[1] Butterworth KT, Prise KM, Verhaegen F. Small animal image-guided radiotherapy: status, considerations and potential for translational impact. Br J Radiol 2015;88:20140634.

[2] Koontz BF, Verhaegen F, De Ruysscher D. Tumour and normal tissue radiobiology in mouse models: how close are mice to mini-humans? $\mathrm{Br} \mathrm{J}$ Radiol 2017;90:20160441.

[3] Tillner F, Thute P, Butof R, Krause M, Enghardt W. Pre-clinical research in small animals using radiotherapy technology-a bidirectional translational approach. Zeitschrift fur medizinische Physik 2014;24:335-51.

[4] Verhaegen F, Granton P, Tryggestad E. Small animal radiotherapy research platforms. Phys Med Biol 2011;56:R55-83.

[5] Rodriguez M, Zhou H, Keall P, Graves E. Commissioning of a novel microCT/RT system for small animal conformal radiotherapy. Phys Med Biol 2009;54:3727-40.

[6] Tryggestad E, Armour M, Iordachita I, Verhaegen F, Wong JW. A comprehensive system for dosimetric commissioning and Monte Carlo validation for the small animal radiation research platform. Phys Med Biol 2009;54:5341-57. 
[7] Lindsay PE, Granton PV, Gasparini A, Jelveh S, Clarkson R, van Hoof S, et al Multi-institutional dosimetric and geometric commissioning of image-guided small animal irradiators. Med Phys 2014;41:031714.

[8] Deng H, Kennedy CW, Armour E, Tryggestad E, Ford E, McNutt T, et al. The small-animal radiation research platform (SARRP): dosimetry of a focused lens system. Phys Med Biol 2007;52:2729-40.

[9] Matinfar M, Iordachita I, Ford E, Wong J, Kazanzides P. Precision radiotherapy for small animal research. Medical image computing and computer-assisted intervention: MICCAI international conference on medical image computing and computer-assisted intervention; 2008;11:619-26.

[10] Wong J, Armour E, Kazanzides P, Iordachita I, Tryggestad E, Deng H, et al. Highresolution, small animal radiation research platform with X-ray tomographic guidance capabilities. Int J Radiat Oncol Biol Phys 2008;71:1591-9.

[11] Clarkson R, Lindsay PE, Ansell S, Wilson G, Jelveh S, Hill RP, et al Characterization of image quality and image-guidance performance of a preclinical microirradiator. Med Phys 2011;38:845-56.

[12] Stojadinovic S, Low DA, Hope AJ, Vicic M, Deasy JO, Cui J, et al. MicroRT-small animal conformal irradiator. Med Phys 2007;34:4706-16.

[13] Graves EE, Zhou H, Chatterjee R, Keall PJ, Gambhir SS, Contag CH, et al. Design and evaluation of a variable aperture collimator for conformal radiotherapy of small animals using a microCT scanner. Med Phys 2007;34:4359-67.

[14] Jensen MD, Hrinivich WT, Jung JA, Holdsworth DW, Drangova M, Chen J, et al. Implementation and commissioning of an integrated micro-CTRT system with computerized independent jaw collimation. Med Phys 2013;40:081706.

[15] Song KH, Pidikiti R, Stojadinovic S, Speiser M, Seliounine S, Saha D, et al. An Xray image guidance system for small animal stereotactic irradiation. Phys Med Biol 2010;55:7345-62.

[16] Wang S, Calderon X, Peng R, Schreiber EC, Zhou O, Chang S. A carbon nanotube field emission multipixel x-ray array source for microradiotherapy application. Appl Phys Lett 2011;98:213701.

[17] Tillner F, Thute P, Lock S, Dietrich A, Fursov A, Haase R, et al. Precise imageguided irradiation of small animals: a flexible non-profit platform. Phys Med Biol 2016:61:3084-108.

[18] Ma CM, Coffey CW, DeWerd LA, Liu C, Nath R, Seltzer SM, et al. AAPM protocol for 40-300 kV X-ray beam dosimetry in radiotherapy and radiobiology. Med Phys 2001;28:868-93.

[19] The IPEMB code of practice for the determination of absorbed dose for X-rays below $300 \mathrm{kV}$ generating potential $(0.035 \mathrm{~mm}$ Al-4 mm Cu HVL; $10-300 \mathrm{kV}$ generating potential), Institution of Physics and Engineering in Medicine and Biology. Physics in Medicine and Biology; 1996; 41: 2605-2625.

[20] Aukett RJ, Burns JE, Greener AG, Harrison RM, Moretti C, Nahum AE, et al. Addendum to the IPEMB code of practice for the determination of absorbed dose for X-rays below $300 \mathrm{kV}$ generating potential $(0.035 \mathrm{~mm} \mathrm{Al}-4 \mathrm{~mm} \mathrm{Cu}$ HVL). Phys Med Biol 2005;50:2739-48.

[21] Granton PV, Podesta M, Landry G, Nijsten S, Bootsma G, Verhaegen F. A combined dose calculation and verification method for a small animal precision irradiator based on onboard imaging. Med Phys 2012;39:4155-66.

[22] Hill MA. The variation in biological effectiveness of X-rays and gamma rays with energy. Radiat Protect Dosim 2004;112:471-81.

[23] Fowler JF. 21 years of biologically effective dose. Br J Radiol 2010;83:554-68.

[24] Desrosiers M, DeWerd L, Deye J, Lindsay P, Murphy MK, Mitch M, et al. The importance of dosimetry standardization in radiobiology. J Res Natl Inst Standard Technol 2013;118:403-18.

[25] Pedersen KH, Kunugi KA, Hammer CG, Culberson WS, DeWerd LA. Radiation biology irradiator dose verification survey. Radiat Res 2016;185:163-8.

[26] Liu FF, workshop p, Okunieff P, Bernhard EJ, Stone HB, Yoo S, et al. Lessons learned from radiation oncology clinical trials. Clin Cancer Res 2013;19:6089-100.

[27] Verhaegen F, van Hoof S, Granton PV, Trani D. A review of treatment planning for precision image-guided photon beam pre-clinical animal radiation studies. Zeitschrift fur medizinische Physik 2014;24:323-34.

[28] Jacques R, Taylor R, Wong J, McNutt T. Towards real-time radiation therapy: GPU accelerated superposition/convolution. Comput Methods Programs Biomed 2010;98:285-92.

[29] Jacques R, Wong J, Taylor R, McNutt T. Real-time dose computation: GPUaccelerated source modeling and superposition/convolution. Med Phys 2011;38:294-305.

[30] Noblet C, Chiavassa S, Smekens F, Sarrut D, Passal V, Suhard J, et al. Validation of fast Monte Carlo dose calculation in small animal radiotherapy with EBT3 radiochromic films. Phys Med Biol 2016;61:3521-35.

[31] Smekens F, Letang JM, Noblet C, Chiavassa S, Delpon G, Freud N, et al. Split exponential track length estimator for Monte-Carlo simulations of smallanimal radiation therapy. Phys Med Biol 2014:59:7703-15.

[32] Cyran CC, Paprottka PM, Eisenblatter M, Clevert DA, Rist C, Nikolaou K, et al. Visualization, imaging and new preclinical diagnostics in radiation oncology. Radiat Oncol 2014:9:3.

[33] Granton PV, Verhaegen F. On the use of an analytic source model for dose calculations in precision image-guided small animal radiotherapy. Phys Med Biol 2013;58:3377-95.

[34] Landry G, Granton PV, Reniers B, Ollers MC, Beaulieu L, Wildberger JE, et al Simulation study on potential accuracy gains from dual energy CT tissue segmentation for low-energy brachytherapy Monte Carlo dose calculations. Phys Med Biol 2011;56:6257-78.

[35] Landry G, Reniers B, Granton PV, van Rooijen B, Beaulieu L, Wildberger JE, et al. Extracting atomic numbers and electron densities from a dual source dual energy CT scanner: experiments and a simulation model. Radiotherapy Oncol 2011;100:375-9.
[36] Bazalova M, Graves EE. The importance of tissue segmentation for dose calculations for kilovoltage radiation therapy. Med Phys 2011:38:3039-49.

[37] Balvert M, van Hoof SJ, Granton PV, Trani D, den Hertog D, Hoffmann AL, et al. A framework for inverse planning of beam-on times for 3D small animal radiotherapy using interactive multi-objective optimisation. Phys Med Biol 2015;60:5681-98.

[38] Stewart JM, Lindsay PE, Jaffray DA. Two-dimensional inverse planning and delivery with a preclinical image guided microirradiator. Med Phys 2013;40:101709.

[39] Hill MA, Vojnovic B. Implications of respiratory motion for small animal image-guided radiotherapy. Br J Radiol 2017;90:20160482.

[40] Pinter C, Lasso A, Wang A, Jaffray D, Fichtinger G. SlicerRT: radiation therapy research toolkit for 3D Slicer. Med Phys 2012;39:6332-8.

[41] van Hoof SJ, Granton PV, Verhaegen F. Development and validation of a treatment planning system for small animal radiotherapy: SmART-Plan. Radiotherapy Oncol 2013;109:361-6.

[42] Osborne DR, Kuntner C, Berr S, Stout D. Guidance for efficient small animal imaging quality control. Mol Imag Biol MIB 2016.

[43] Kersemans V, Thompson J, Cornelissen B, Woodcock M, Allen PD, Buls N, et al. Micro-CT for anatomic referencing in PET and SPECT: radiation dose, biologic damage, and image quality. J Nucl Med 2011;52:1827-33.

[44] Sharma S, Narayanasamy G, Przybyla B, Webber J, Boerma M, Clarkson R, et al. Advanced small animal conformal radiation therapy device. Technol Cancer Res Treat 2017; 16:45-56.

[45] Feldkamp LA, Davis LC, Kress JW. Practical cone-beam algorithm. Journal of the Optical Society of America A. 1984:1:612-9.

[46] Du LY, Umoh J, Nikolov HN, Pollmann SI, Lee TY, Holdsworth DW. A quality assurance phantom for the performance evaluation of volumetric micro-CT systems. Phys Med Biol 2007;52:7087-108.

[47] Ford NL, Thornton MM, Holdsworth DW. Fundamental image quality limits for microcomputed tomography in small animals. Med Phys 2003;30:2869-77.

[48] Chavarrias C, Vaquero JJ, Sisniega A, Rodriguez-Ruano A, Soto-Montenegro ML, Garcia-Barreno P, et al. Extraction of the respiratory signal from small-animal CT projections for a retrospective gating method. Phys Med Biol 2008;53:4683-95.

[49] Schyns LE, Almeida IP, van Hoof SJ, Descamps B, Vanhove C, Landry G, et al. Optimizing dual energy cone beam CT protocols for preclinical imaging and radiation research. Br J Radiol 2017;90:20160480.

[50] Franc BL, Acton PD, Mari C, Hasegawa BH. Small-animal SPECT and SPECT/CT: important tools for preclinical investigation. J Nucl Med 2008;49:1651-63.

[51] Rowland DJ, Cherry SR. Small-animal preclinical nuclear medicine instrumentation and methodology. Semin Nucl Med 2008;38:209-22.

[52] Kagadis GC, Loudos G, Katsanos K, Langer SG, Nikiforidis GC. In vivo small animal imaging: current status and future prospects. Med Phys 2010;37:6421-42.

[53] Vanhove C, Bankstahl JP, Kramer SD, Visser E, Belcari N, Vandenberghe S. Accurate molecular imaging of small animals taking into account animal models, handling, anaesthesia, quality control and imaging system performance. EJNMMI Phys 2015;2:31.

[54] Goertzen AL, Bao Q, Bergeron M, Blankemeyer E, Blinder S, Canadas M, et al. NEMA NU 4-2008 comparison of preclinical PET imaging systems. J Nucl Med 2012;53:1300-9.

[55] Soret M, Bacharach SL, Buvat I. Partial-volume effect in PET tumor imaging. J Nucl Med 2007; 48:932-45.

[56] Bolcaen J, Descamps B, Deblaere K, Boterberg T, Hallaert G, Van den Broecke C, et al. MRI-guided 3D conformal arc micro-irradiation of a F98 glioblastoma rat model using the Small Animal Radiation Research Platform (SARRP). J Neurooncol 2014;120:257-66.

[57] Gutierrez S, Descamps B, Vanhove C. MRI-only based radiotherapy treatment planning for the rat brain on a small animal radiation research platform (SARRP). PloS One 2015;10:e0143821.

[58] Kim H, Hartman YE, Zhai G, Chung TK, Korb ML, Beasley TM, et al. Dynamic contrast-enhanced MRI evaluates the early response of human head and neck tumor xenografts following anti-EMMPRIN therapy with cisplatin or irradiation. J Magnet Reson Imag JMRI 2015;42:936-45.

[59] Doblas S, Almeida GS, Ble FX, Garteiser P, Hoff BA, McIntyre DJ, et al. Apparent diffusion coefficient is highly reproducible on preclinical imaging systems: evidence from a seven-center multivendor study. J Magnet Reson Imag JMRI 2015:42:1759-64.

[60] Frenzel T, Kaul MG, Ernst TM, Salamon J, Jackel M, Schumacher U, et al. Magnetic resonance imaging for precise radiotherapy of small laboratory animals. Zeitschrift fur medizinische Physik 2017:27:6-12.

[61] Lee YC, Fullerton GD, Baiu C, Lescrenier MG, Goins BA. Preclinical multimodality phantom design for quality assurance of tumor size measurement. BMC Med Phys 2011:11:1.

[62] O'Callaghan J, Wells J, Richardson S, Holmes H, Yu Y, Walker-Samuel S, et al. Is your system calibrated? MRI gradient system calibration for pre-clinical, highresolution imaging. PloS One 2014;9:e96568.

[63] Wiant D, Atwood TF, Olson J, Papagikos M, Forbes ME, Riddle DR, et al. Gamma knife radiosurgery treatment planning for small animals using high-resolution 7T micro-magnetic resonance imaging. Radiat Res 2009;172:625-31.

[64] Tuli R, Armour M, Surmak A, Reyes J, Iordachita I, Patterson M, et al. Accuracy of off-line bioluminescence imaging to localize targets in preclinical radiation research. Radiat Res 2013;179:416-21.

[65] Tuli R, Surmak A, Reyes J, Hacker-Prietz A, Armour M, Leubner A, et al. Development of a novel preclinical pancreatic cancer research model: 
bioluminescence image-guided focal irradiation and tumor monitoring of orthotopic xenografts. Transl Oncol 2012;5:77-84.

[66] Yu J, Zhang B, Iordachita II, Reyes J, Lu Z, Brock MV, et al. Systematic study of target localization for bioluminescence tomography guided radiation therapy. Med Phys 2016;43:2619.

[67] Dehghani H, Davis SC, Jiang S, Pogue BW, Paulsen KD, Patterson MS. Spectrally resolved bioluminescence optical tomography. Optics Lett 2006;31:365-7.

[68] Negrin RS, Contag CH. In vivo imaging using bioluminescence: a tool for probing graft-versus-host disease. Nat Rev Immunol 2006;6:484-90.

[69] Dehghani H, Davis SC, Pogue BW. Spectrally resolved bioluminescence tomography using the reciprocity approach. Med Phys 2008;35:4863-71.

[70] Virostko J, Powers AC, Jansen ED. Validation of luminescent source reconstruction using single-view spectrally resolved bioluminescence images. Appl Optics 2007;46:2540-7.

[71] Weersink RA, Ansell S, Wang A, Wilson G, Shah D, Lindsay PE, et al. Integration of optical imaging with a small animal irradiator. Med Phys 2014;41:102701.

[72] Jacques SL. Optical properties of biological tissues: a review. Phys Med Biol 2013;58:R37-61.

[73] Arridge SR. Optical tomography in medical imaging. Inverse ProbInverse Problems 1999;15:R41-93.

[74] Klose AD, Beattie BJ, Dehghani H, Vider L, Le C, Ponomarev V, et al. In vivo bioluminescence tomography with a blocking-off finite-difference SP3 method and MRI/CT coregistration. Med Phys 2010;37:329-38.

[75] Naser MA, Patterson MS, Wong JW. Self-calibrated algorithms for diffuse optical tomography and bioluminescence tomography using relative transmission images. Biomed Opt Express 2012:3:2794-808.

[76] Zhang B, Wang KK, Yu J, Eslami S, Iordachita I, Reyes J, et al. Bioluminescence tomography-guided radiation therapy for preclinical research. Int J Radiat Oncol Biol Phys 2016;94:1144-53.

[77] Wang L, Jacques SL, Zheng L. MCML-Monte Carlo modeling of light transport in multi-layered tissues. Comput Methods Programs Biomed 1995;47:131-46.

[78] Zhu C, Liu Q. Review of Monte Carlo modeling of light transport in tissues. J Biomed Opt 2013:18:50902.

[79] Darne C, Lu Y, Sevick-Muraca EM. Small animal fluorescence and bioluminescence tomography: a review of approaches, algorithms and technology update. Phys Med Biol 2014;59:R1-R64.
[80] Dehghani H, Eames ME, Yalavarthy PK, Davis SC, Srinivasan S, Carpenter CM, et al. Near infrared optical tomography using NIRFAST: Algorithm for numerical model and image reconstruction. Commun Numer Meth En 2009;25:711-32.

[81] Naser MA, Patterson MS, Wong JW. Algorithm for localized adaptive diffuse optical tomography and its application in bioluminescence tomography. Phys Med Biol 2014;59:2089-109.

[82] Dogdas B, Stout D, Chatziioannou AF, Leahy RM. Digimouse: a 3D whole body mouse atlas from CT and cryosection data. Phys Med Biol 2007;52:577-87.

[83] Granton PV, Dubois L, van Elmpt W, van Hoof SJ, Lieuwes NG, De Ruysscher D, et al. A longitudinal evaluation of partial lung irradiation in mice by using a dedicated image-guided small animal irradiator. Int J Radiat Oncol Biol Phys. 2014;90:696-704.

[84] http://www.dicom.nema.org.

[85] ftp://medical.nema.org/medical/dicom/final/sup187_ft_ preclinicalanimalacquisitioncontext.pdf.

[86] Peschke P, Karger CP, Scholz M, Debus J, Huber PE. Relative biological effectiveness of carbon ions for local tumor control of a radioresistant prostate carcinoma in the rat. Int J Radiat Oncol Biol Phys 2011;79:239-46.

[87] Saager M, Glowa C, Peschke P, Brons S, Scholz M, Huber PE, et al. Carbon ion irradiation of the rat spinal cord: dependence of the relative biological effectiveness on linear energy transfer. Int J Radiat Oncol Biol Phys 2014;90:63-70.

[88] Greubel C, Assmann W, Burgdorf C, Dollinger G, Du G, Hable V, et al. Scanning irradiation device for mice in vivo with pulsed and continuous proton beams, Radiat Environ Biophys 2011;50:339-44.

[89] Girst S, Greubel C, Reindl J, Siebenwirth C, Zlobinskaya O, Walsh DW, et al. Proton Minibeam Radiation Therapy Reduces Side Effects in an In Vivo Mouse Ear Model. Int J Radiat Oncol Biol Phys 2016;95:234-41.

[90] Ford E, Emery R, Huff D, Narayanan M, Schwartz J, Cao N, et al. An imageguided precision proton radiation platform for preclinical in vivo research. Phys Med Biol 2017;62:43-58.

[91] van der Heyden B, van Hoof S, Schyns L, Verhaegen F. The influence of respiratory motion on dose delivery in a mouse lung tumor irradiation using the 4D MOBY phantom. Br J Radiol 2017;90. 20160419. 